\title{
Comparison of DWIBS/T2 image fusion and PET/CT for the diagnosis of cancer in the abdominal cavity
}

\author{
MINORU TOMIZAWA ${ }^{1}$, FUMINOBU SHINOZAKI ${ }^{2}$, YOSHITAKA UCHIDA ${ }^{3}$, KATSUHIRO UCHIYAMA ${ }^{3}$, \\ SATOMI TANAKA $^{2}$, TAKAFUMI SUNAOSHI ${ }^{2}$, DAISUKE KANO ${ }^{2}$, ERIKO SUGIYAMA ${ }^{2}$, MISAKI SHITE ${ }^{2}$, \\ RYOUTA HAGA $^{2}$, YOSHIYA FUKAMIZU ${ }^{2}$, TOSHIYUKI FUJITA ${ }^{2}$, SATOSHI KAGAYAMA ${ }^{2}$, \\ RUMIKO HASEGAWA ${ }^{4}$, YOSHINORI SHIRAI ${ }^{4}$, YASUFUMI MOTOYOSHI ${ }^{5}$, \\ TAKAO SUGIYAMA $^{6}$, SHIGENORI YAMAMOTO ${ }^{7}$ and NAOKI ISHIGE $^{8}$
}

\begin{abstract}
Departments of ${ }^{1}$ Gastroenterology and ${ }^{2}$ Radiology, National Hospital Organization Shimoshizu Hospital, Yotsukaido, Chiba 284-0003; ${ }^{3}$ Diagnostic PET Imaging Center, Department of Radiology, Sannoh Hospital Medical Center, Chiba 263-0002; Departments of ${ }^{4}$ Surgery, ${ }^{5}$ Neurology, ${ }^{6}$ Rheumatology, ${ }^{7}$ Pediatrics and

${ }^{8}$ Neurosurgery, National Hospital Organization Shimoshizu Hospital, Yotsukaido, Chiba 284-0003, Japan
\end{abstract}

Received January 24, 2016; Accepted February 1, 2017

DOI: $10.3892 /$ etm.2017.4987

\begin{abstract}
Fusion images of diffusion-weighted whole-body imaging with background body signal suppression and T2-weighted image (DWIBS/T2) demonstrate a strong signal for malignancies, with a high contrast against the surrounding tissues, and enable anatomical analysis. In the present study, DWIBS/T2 was compared with ${ }^{18} \mathrm{~F}$-fluorodeoxyglucose $\left({ }^{18} \mathrm{~F}-\mathrm{FDG}\right)$ positron emission tomography/computed tomography (PET/CT) for diagnosing cancer in the abdomen. Patient records, including imaging results of examination conducted between November 2012 and May 2014, were analyzed retrospectively. In total, 10 men (age, 73.6 \pm 9.6 years) and 8 women (age, 68.9 \pm 7.1 years) were enrolled into the current study. Of the enrolled patients, 2 were diagnosed with hepatocellular carcinoma, 1 with cholangiocellular carcinoma, 1 with liver metastasis, 2 with pancreatic ductal adenocarcinoma, 1 with renal cell carcinoma and 1 with malignant lymphoma. Benign lesions were also analyzed, including adenomyomatosis of the gallbladder (5 patients), intraductal papillary mucinous neoplasm (4 patients) and right adrenal adenoma (1 case). All the patients with cancer showed positive results on DWIBS/T2 images. However, only 7 out of 8 patients were positive with $\mathrm{PET} / \mathrm{CT}$. One patient with right renal cellular carcinoma was positive with DWIBS/T2, but negative with PET/CT. All the patients with benign lesions were negative with DWIBS/T2 and PET/CT. In conclusion, DWIBS/T2 was more sensitive in
\end{abstract}

Correspondence to: Dr Minoru Tomizawa, Department of Gastroenterology, National Hospital Organization Shimoshizu Hospital, 934-5 Shikawatashi, Yotsukaido, Chiba 284-0003, Japan E-mail: nihminor-cib@umin.ac.jp

Key words: computed tomography, endoscopic ultrasonography, pancreatic ductal adenocarcinoma, adenomyomatosis of the gallbladder, intraductal papillary mucinous neoplasm diagnosing cancer of organs in the abdominal cavity compared with PET/CT. Furthermore, negative results with DWIBS/T2 and PET/CT were useful for the diagnosis of benign lesions, such as adenomyomatosis of the gallbladder and intraductal papillary mucinous neoplasm.

\section{Introduction}

The abdominal cavity harbors solid organs, such as the liver, pancreas and kidneys. The liver is a common site of primary cancer, such as hepatocellular carcinoma (HCC) and cholangiocellular carcinoma (CCC), as well as of metastatic cancer (1). Renal cell carcinoma (RCC) can form in the kidney, while malignant lymphoma develops in the lymph nodes in the abdominal cavity. In addition, pancreatic ductal adenocarcinoma (PDA) occurs in the pancreas, and has a poor prognosis despite advances in treatment strategies, such as surgery, irradiation and chemotherapy (2-4). Furthermore, intraductal papillary mucinous neoplasm (IPMN) forms multiple cystic lesions in the pancreas (5), and may then result in intraductal papillary mucinous adenocarcinoma (6). In order to improve the prognosis of these diseases, early detection of pancreatic cancer is important (7).

${ }^{18} \mathrm{~F}$-fluorodeoxyglucose $\left({ }^{18} \mathrm{~F}-\mathrm{FDG}\right)$ positron emission tomography/computed tomography (PET/CT) takes advantage of the Warburg effect (8), which suggests that cancer cells require more glucose than normal cells due to the activation of glycolysis. Therefore, cancer cells readily take up ${ }^{18} \mathrm{~F}-\mathrm{FDG}$, an analogue of glucose, which is then not metabolized and accumulates inside these cells to a much greater extent than in non-cancerous cells. Subsequently, PET/CT imaging is used to detect the signal of ${ }^{18} \mathrm{~F}-\mathrm{FDG}$ and construct images of its uptake pattern. PET/CT has been established as a useful imaging modality for cancer diagnosis (9), and serves plays an important role in the evaluation of TMN staging of cancer and its metastasis to lymph nodes and distant organs $(10,11)$. However, a possible disadvantage of 
$\mathrm{PET} / \mathrm{CT}$ is that the patient is exposed to ionizing radiation from ${ }^{18} \mathrm{~F}-\mathrm{FDG}$.

Diffusion-weighted whole body imaging with background body-signal suppression (DWIBS) is an imaging technique based on images that are created through analysis of the random movement of water at a molecular level (Brownian motion) and its diffusion through the tissues $(12,13)$. Dense, highly cellular tumor tissue suppresses water diffusion, creating a strong DWIBS signal. DWIBS images are acquired with multiple-signal averaging, pre-pulse fat suppression and heavy diffusion weighting during free breathing (14).

One of the advantages of DWIBS is the strong contrast of positive signals against the surrounding tissues, thus DWIBS is useful for the detection and staging of cancer (15). However, a major limitation of DWIBS is that it is occasionally difficult to evaluate the positive signals in the context of the anatomical setting since the surrounding tissues are hard to observe due to the suppression of their signals. Fusion images can be created by overlapping DWIBS onto T2-weighted images (T2WI; DWIB/T2) (15-17). The image fusion is performed on a workstation, and DWIBS/T2 clearly enables functional analysis of DWIBS signals in the anatomical setting.

In the present study, the accuracy and clinical utility of DWIBS/T2 and PET/CT imaging for patients with cancer in the solid organs of the abdominal cavity were retrospectively compared.

\section{Materials and methods}

Ethical statement. The present study was approved by the Ethics Committee of the National Hospital Organization Shimoshizu Hospital (Yotsukaido, China). This study was not considered to be a clinical trial, as the procedures were performed as a part of routine clinical practice. Written informed consent for inclusion into the study was thus waived. Patient records were anonymized and retrospectively analyzed. Written informed consent was obtained from all patients who were subjected to DWIBS/T2, PET/CT, magnetic resonance cholangiopancreatography, endoscopic ultrasonography (US) and ultrasound-guided biopsy.

Diagnostic procedure. Abdominal ultrasonography and contrast-enhanced CT were typically performed for patients suspected of cancer in their abdominal cavity. Certain patients were also subjected to DWIBS/T2 to clarify the extent of cancer (15). PET/CT was performed in patients in which diagnosis was difficult with the aforementioned diagnostic imaging techniques. PET/CT was also performed to assess the staging of cancer. These results were compared with follow-up information, as a number of patients were assessed by histological analysis. Histological diagnosis was determined with surgical specimens or US-guided biopsy.

Inclusion criteria. Patients were enrolled into the present study when they met the following criteria: i) Suspected with cancer of the abdominal cavity, such as liver, pancreas or kidney cancer; ii) DWIBS/T2 results were available; and iii) PET/CT images were available. For patients referred to another hospital for the management of cancer, enrollment into the study was restricted to those whose reply was received to clarify their final diagnosis. Patients were also enrolled when their final diagnosis was a benign lesion in order to evaluate the positivity of DWIBS/T2 and PET/CT for benign lesions.

Exclusion criteria. Patients were excluded when DWIBS/T2 or PET/CT results were not available. In addition, patients were excluded when they were diagnosed with alimentary tract cancer.

Study design. Patient records, including imaging results from patients managed in the National Hospital Organization Shimoshizu Hospital between November 2012 and May 2014, were analyzed retrospectively. The enrolled patients were subjected to both DWIBS/T2 and PET/CT, and diagnosed with malignant or benign lesions in the abdominal cavity with imaging diagnostics or pathological specimens obtained by surgery or US-guided biopsy. All patients with benign lesions were regularly followed-up with abdominal US and CT with or without contrast-enhancement in our hospital.

Magnetic resonance imaging (MRI). All MRI studies were performed using a 1.5 Tesla scanner (Achieva, software version 3.2.2; Philips Medical Systems, Best, The Netherlands). T1-weighted, T2-weighted and diffusion-weighted images (DWIs) were obtained with pulse sequences as depicted in Table I. DWIBS/T2 images were constructed with an Extended MR WorkSpace (Philips Medical Systems). The DWI gradients were applied along the $\mathrm{x}, \mathrm{y}$ and $\mathrm{z}$-axes before and after a $180^{\circ}$ inversion pre-pulse to obtain fat-saturated, isotropic images with DWI sensitivity. The following parameters were used for a single stack: b-values, 0 and $800 \mathrm{~mm}^{2} / \mathrm{s}$; repetition time, $6960 \mathrm{msec}$; echo time, $79 \mathrm{msec}$; inversion recovery, $150 \mathrm{msec}$; acquisition matrix, 176x115; reconstruction matrix, 256; field of view, right/left at $530 \mathrm{~mm}$, anterior/posterior at $349 \mathrm{~mm}$, and feet/head at $226 \mathrm{~mm}$; slice thickness, $6 \mathrm{~mm}$; size of reconstructed voxel, 2.07x 2.08x6 $\mathrm{mm}^{3}$; four averages. A radiologist and a gastroenterologist analyzed the DWIBS/T2 images. To rule out the $\mathrm{T} 2$ shine-through effect or to differentiate malignant lesions from non-malignant causes of restricted diffusion, a decreased signal on the apparent diffusion coefficient (ADC) to determine a 'positive ADC map' (18). It took $20 \mathrm{~min}$ to perform both the DWIBS and T2-weighted imaging together.

PET/CT imaging. PET/CT was examined in Sannoh Hospital Medical Center. Patients were subjected to PET/CT in the Diagnostic PET Imaging Center, Department of Radiology, Sannoh Medical Center (Chiba, Japan) (19). Patients fasted overnight or for at least $6 \mathrm{~h}$ before injection of 4.0 mega Bq/kg of ${ }^{18} \mathrm{~F}-\mathrm{FDG}$. At $60 \mathrm{~min}$ after the injection, images were acquired. Patients rested during the uptake of ${ }^{18} \mathrm{~F}-\mathrm{FDG}$ to minimize non-specific uptake in muscles. An integrated PET/CT (Discovery ST; GE Medical Systems, Milwaukee, WI, USA) was used for image acquisition. CT scanning was performed with $120 \mathrm{kV}, 25 \mathrm{~mA}$, a pitch of 1.75 , a section thickness of $3.3 \mathrm{~mm}$, a field of view of $50 \mathrm{~cm}$, and a matrix size of 512x512. Immediately after the unenhanced CT scan, a PET scan was performed with a $3.3-\mathrm{mm}$ thick section, a matrix size of $128 \times 128$, and an acquisition time of $2.5 \mathrm{~min}$. PET data sets were reconstructed iteratively with an ordered subsets expectation maximization algorithm and segmented 
Table I. Pulse sequences used in the present study.

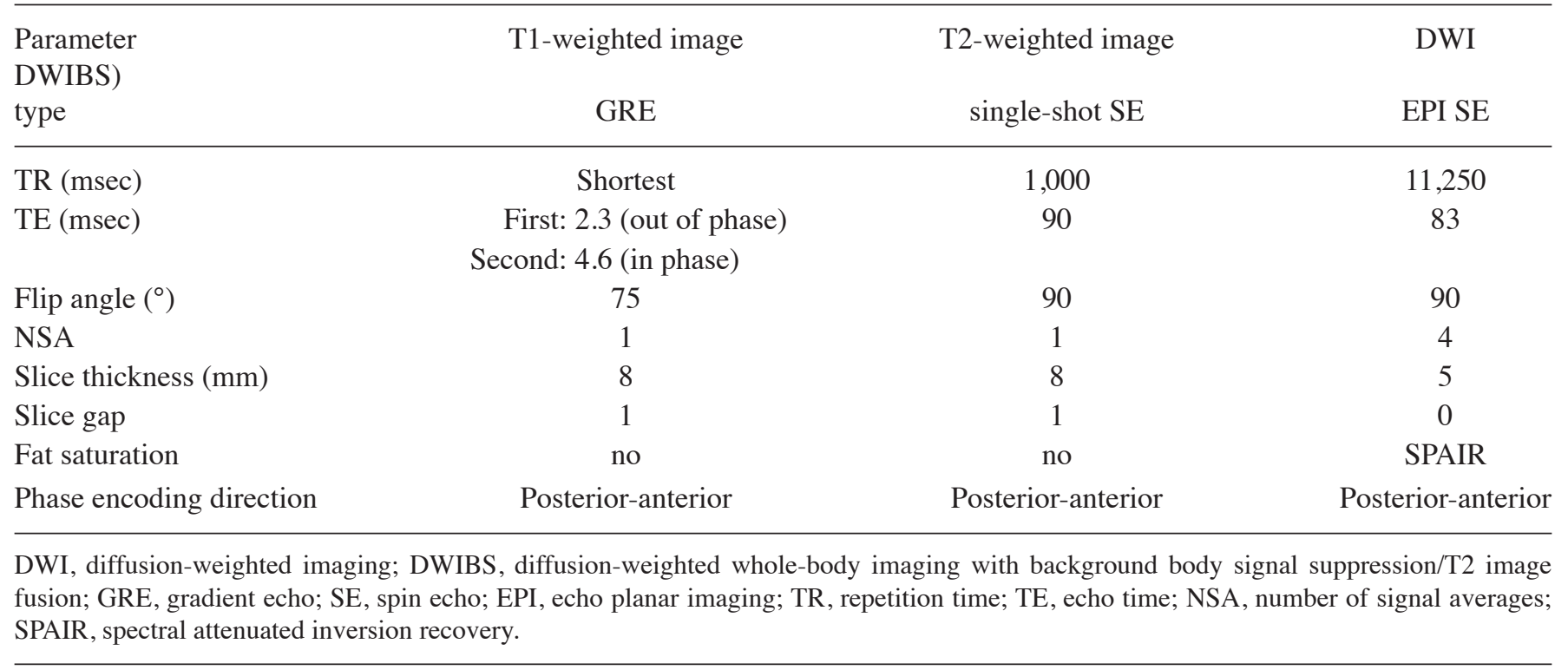

attenuation correction (three iterations, 15 subsets) and fused onto the CT data. The fused image of PET and CT allowed the evaluation of signals from PET in the anatomical settings. The standardized uptake value $(\mathrm{SUV}>2.5)$ was calculated as previously described (20), using the following formula: SUV $(\mathrm{g} / \mathrm{ml})=$ regional radioactivity concentration $(\mathrm{Bq} / \mathrm{ml}) /[$ injected dose $(\mathrm{Bq}) /$ body weight $(\mathrm{g})]$.

CT imaging. In National Hospital Organization Shimoshizu Hospital, CT was performed using a 16-detector row CT scanner (SOMATOM Emotion 16; Siemens Healthcare, Munich, Germany). Imaging parameters for three-phase contrast-enhanced images were as follows: Tube voltage, $130 \mathrm{kVp}$; gantry rotation speed, 0.6 rotations/sec; maximum allowable tube current, $120 \mathrm{~mA}$. For certain patients, a contrast medium was administered intravenously (100 $\mathrm{ml}$ iopamidol at $3 \mathrm{ml} / \mathrm{sec}$; Konica Minolta Healthcare, Tokyo, Japan). CT images were acquired prior to injection of the contrast medium and at 30, 70 and $180 \mathrm{sec}$ after injection.

Abdominal and endoscopic US. Abdominal US was performed by senior fellows of the Japan Society of Ultrasonics in Medicine (Tokyo, Japan; www.jsum.or.jp) in the US unit. The device used was an SSA-700A system (Toshiba Medical Systems Corp., Ohtawara, Japan). The probes used were a 3.75-MHz curved-array probe (PVT-375BT) or a $3-\mathrm{MHz}$ sector-array probe (PST-308T; both from Toshiba Medical Systems Corp.). For patients with PDA, adenomyomatosis or IPMN, endoscopic US was also performed with a GF-UCT260 ultrasound gastrovideoscope (Olympus Corp., Tokyo, Japan) by senior fellows of the Japan Gastroenterological Endoscopy Society (www.jges.net).

\section{Results}

Patients. A total of 10 males (age, $73.6 \pm 9.6$ years) and 8

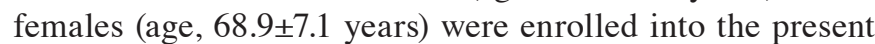
study. Of the enrolled patients, 8 were finally diagnosed with malignant lesions, including 2 patients with $\mathrm{HCC}, 1$ with $\mathrm{CCC}$, 1 with metastatic liver cancer, 2 with PDA, 1 with RCC and 1 with malignant lymphoma surrounding the abdominal aorta. The remaining patients were diagnosed with benign lesions, which included 5 cases of adenomyomatosis of the gallbladder, 4 cases of IPMN and 1 case of right adrenal adenoma.

Diagnostic performance evaluation. To evaluate the diagnostic performance of DWIBS/T2, detection of cancer in the abdominal cavity was compared with the diagnostic ability of PET/CT (Table II). Positive results were obtained using DWIBS/T2 in all patients with malignancies. By contrast, only 7 out of 8 patients were positively diagnosed with PET/CT. These results suggest that DWIBS/T2 was more sensitive in the detection of cancer of the abdominal cavity.

Representative cases of PDA diagnosis. Images of PDA are demonstrated in Fig. 1, since the precise diagnosis of this cancer is important for patient management, but is occasionally difficult to diagnose. PDA was detected as a low echo lesion with abdominal US (Fig. 1A) and as an unenhanced area with contrast-enhanced CT (Fig. 1B). PDA was not clearly observed by T2WI (Fig. 1C). However, the contrast of PDA tissues was significantly higher when compared with the background tissues on DWIBS (Fig. 1D). Furthermore, DWIBS/T2 illustrated a high signal on the head of the pancreas (Fig. 1E), and enabled the evaluation of the high intensity area in the anatomical setting. Finally, PDA was also strongly positive with PET/CT (Fig. 1F), although it was difficult to evaluate the extent of PDA with this method. These figures demonstrated that DWIBS/T2 clearly showed the PDA lesion as a significantly high intensity area. It was also demonstrated that it was easier to evaluate the extent of PDA in the anatomical setting using DWIBS/T2 as compared with PET/CT.

RCC diagnosis. Contrast-enhanced CT provided positive results for 1 patient with right RCC (Fig. 2A and B). DWIBS/T2 also demonstrated positive results in this patient (Fig. 2C). 
Table II. Diagnostic performance for malignant lesions.

\begin{tabular}{|c|c|c|c|c|c|}
\hline Patient no. & Sex & Age (years) & Diagnosis & DWIBS/T2 & $\mathrm{PET} / \mathrm{CT}$ \\
\hline 1 & $\mathrm{~F}$ & 73 & $\mathrm{HCC}$ & $(+)$ & $(+)$ \\
\hline 2 & M & 83 & $\mathrm{HCC}$ & $(+)$ & $(+)$ \\
\hline 3 & $\mathrm{~F}$ & 57 & $\mathrm{CCC}$ & $(+)$ & $(+)$ \\
\hline 4 & $\mathrm{~F}$ & 74 & Liver metastasis & $(+)$ & $(+)$ \\
\hline 5 & $\mathrm{~F}$ & 67 & PDA & $(+)$ & $(+)$ \\
\hline 6 & $\mathrm{M}$ & 83 & PDA & $(+)$ & $(+)$ \\
\hline 7 & $\mathrm{~F}$ & 81 & RCC (right) & $(+)$ & $(-)$ \\
\hline 8 & $\mathrm{~F}$ & 67 & ML & $(+)$ & $(+)$ \\
\hline
\end{tabular}

DWIBS/T2, diffusion-weighted whole body imaging with background body signal suppression/T2 weighted image fusion; PET/CT, positron emission tomography/computed tomography; M, male; F, female; (+), positive results; (-), negative results; HCC, hepatocellular carcinoma; CCC, cholangiocellular carcinoma; PDA, pancreas ductal cancer; RCC, renal cellular carcinoma; ML, malignant lymphoma.
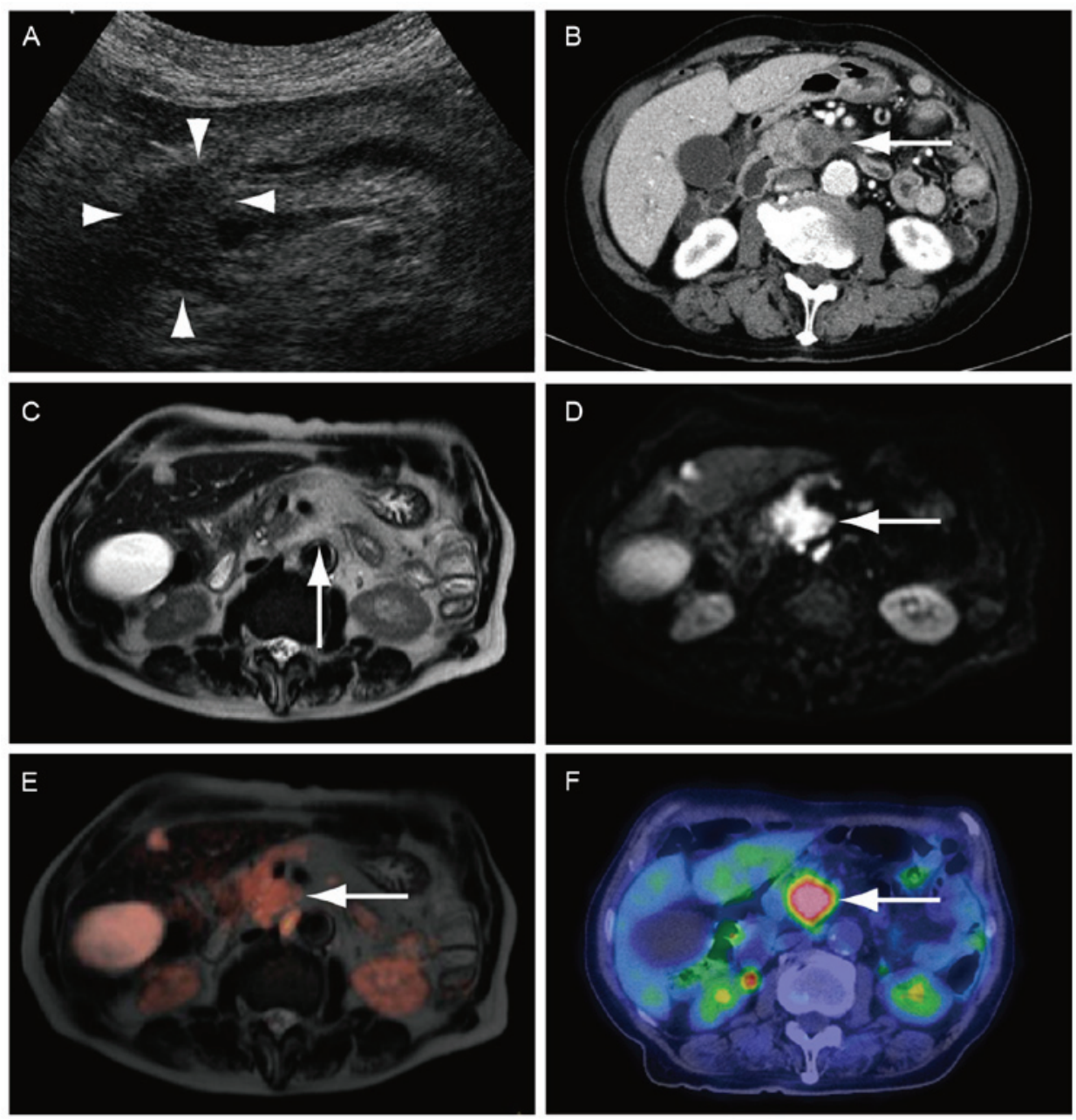

Figure 1. A representative case of pancreatic cancer in an 83-year-old man who presented with back pain. (A) Abdominal ultrasonography illustrates a low echo lesion (arrowheads) in the head of the pancreas. (B) Contrast-enhanced CT image demonstrating an unenhanced area (arrow). (C) T2-weighted image, which does not show an area with different intensity in the pancreas as compared with the surrounding tissues. (D) DWIBS demonstrating a significantly high intensity area (arrow). (E) DWIBS/T2 shows a high intensity area in the head of the pancreas. DWIBS clearly showed the extent of the cancer, while DWIBS/T2 enabled the evaluation of the extent of the cancer in the anatomical settings. (F) PET/CT shows a significantly high signal at the head of the pancreas; however, it was hard to evaluate the extent of the cancer with PET/CT. DWIBS, diffusion-weighted whole body imaging with background body signal suppression; DWIBS/T2, fusion of DWIBS and T2-weighted image; PET, positron emission tomography; CT, computed tomography.

By contrast, PET/CT was negative for RCC (Fig. 2D). These results suggest that $\mathrm{PET} / \mathrm{CT}$ may occasionally give a negative diagnosis in patients with cancer. It was also observed that DWIBS/T2 provided a positive diagnosis for cancer that was 

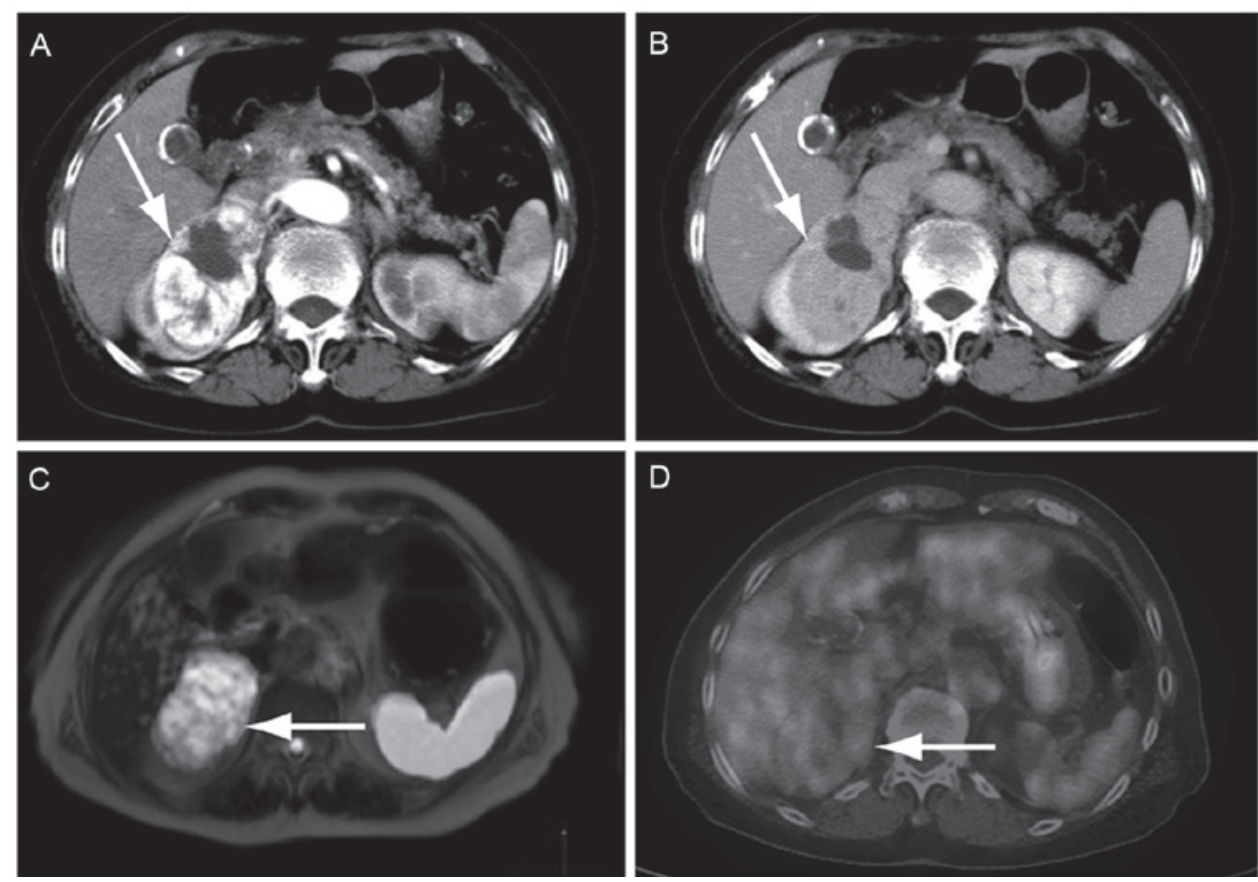

Figure 2. A representative case of renal cell carcinoma in an 81-year-old woman who visited our hospital with back pain and was diagnosed with acute cholangitis. (A) Contrast-enhanced CT image, demonstrating an enhanced tumor (arrow) in the right kidney at 10 sec after the administration of contrast medium. (B) The contrast enhancement agent was washed-out tumor at $180 \mathrm{sec}$. (C) DWIBS/T2 image, demonstrating a high intensity tumor. (D) The tumor is negative on the PET/CT image. DWIBS/T2, diffusion-weighted whole body imaging with background body signal suppression/T2-weighted image fusion; PET, positron emission tomography; CT, computed tomography.

negative with PET/CT. Thus, it is suggested that DWIBS/T2 was more sensitive compared with PET/CT.

Benign lesion evaluation. To evaluate the positivity of the signal of DWIBS/T2 in benign lesions, images were compared with PET/CT (Table III). All the patients with benign lesions had negative results upon DWIBS/T2 and PET/CT imaging (Table III). These results suggested that DWIBS/T2 and $\mathrm{PET} / \mathrm{CT}$ provided negative results for benign lesions.

IPMN was demonstrated as a cystic lesion with magnetic resonance cholangiopancreatography (Fig. 3A). This lesion was further investigated with endoscopic US (Fig. 3B), and was seen as a high intensity signal on a T2WI (Fig. 3C). However, IPMN was negative in DWIBS and DWIBS/T2 (Fig. 3D and E), as well as in PET/CT images (Fig. 3F). These results clearly showed that DWIBS/T2 and PET/CT were negative for IPMN. It is also suggested that DWIBS/T2 enabled the evaluation of the negative results in the anatomical setting.

\section{Discussion}

In order to improve the anatomical analysis, fusion images of DWIBS with other images can be obtained. Manenti et al (21) reported that fusion images of DWIBS and contrast-enhanced CT had the same performance as PET/CT for the detection of primary site tumors and metastasis. As shown in Fig. 1, DWIBS/T2 enabled evaluation of the extent of PDA in the anatomical setting.

Cancer cells exhibit a positive signal with T2WI and DWI (15). However, with T2WI and DWI, positive signals are occasionally unclear, due to strong background signals. DWIBS suppresses these background signals and therefore, cancer is easily detected using DWIBS with a strong positive signal against a low background. Wang et al (22) reported successful detection of HCC and CCC by DWIBS, but not of PDA. In the present study, PDA was successfully detected with DWIBS/T2, as well as PET/CT. The current data suggest that DWIBS/T2 has the same diagnostic performance for PDA as PET/CT. In addition, RCC was negative in PET/CT images in the present study, while it was positive in DWIBS/T2. However, PET/CT has been reported to typically provide a positive diagnosis for RCC (23). The negative results in the present study may be due to the biological characteristics and pathological features of the tumor $(24,25)$. For instance, the glucose metabolism may have been low in the RCC of our patient (26). Notably, DWIBS/T2 provided positive diagnosis for RCC that was negative on PET/CT imaging. These results indicate that DWIBS/T2 was more sensitive for the diagnosis of cancer in the organs of the abdominal cavity.

In the present study, DWBIS/T2 and PET/CT were negative for all of the benign lesions, such as adenomyomatosis of the gallbladder and IPMN. A major issue with adenomyomatosis of the gallbladder is differentiating it from gallbladder cancer (27). Our previous study has already reported that IPMN was negative on DWBIBS/T2 (28). The present study confirmed these negative results with $\mathrm{PET} / \mathrm{CT}$ imaging. These previous and current findings suggest that negative results with DWIBS/T2 or PET/CT may indicate the presence of IPMN.

Overall, the current study results suggest that integrated assessment with DWIBS/T2 and PET/CT is beneficial for patients with cancer in the solid organs in the abdominal cavity (29). However, a major limitation of the study was that it was conducted on a small number of patients. Another limitation was that lymph node metastasis was not evaluated. 
Table III Results of benign lesions.

\begin{tabular}{lcclcc}
\hline Patient no. & Sex & Age (years) & \multicolumn{1}{c}{ Diagnosis } & DWIBS/T2 & PET-CT \\
\hline 1 & M & 60 & Adenomyomatosis & $(-)$ & $(-)$ \\
2 & M & 63 & Adenomyomatosis & $(-)$ & $(-)$ \\
3 & M & 70 & Adenomyomatosis & $(-)$ & $(-)$ \\
4 & F & 65 & Adenomyomatosis of the gallbladder & $(-)$ & $(-)$ \\
5 & M & 71 & Adenomyomatosis of the gallbladder & $(-)$ & $(-)$ \\
6 & M & 85 & IPMN & $(-)$ & $(-)$ \\
7 & M & 73 & IPMN & $(-)$ & $(-)$ \\
8 & M & 64 & IPMN & $(-)$ & $(-)$ \\
9 & F & 67 & IPMN & Adrenal adenoma (right) & $(-)$ \\
10 & M & 64 & & & $(-)$ \\
\hline
\end{tabular}

DWIBS/T2, diffusion-weighted whole body imaging with background body signal suppression/T2 weighted image fusion; PET/CT, positron emission tomography/computed tomography; M, male; F, female; (-), negative results; IPMN, intraductal papillary mucinous neoplasm.
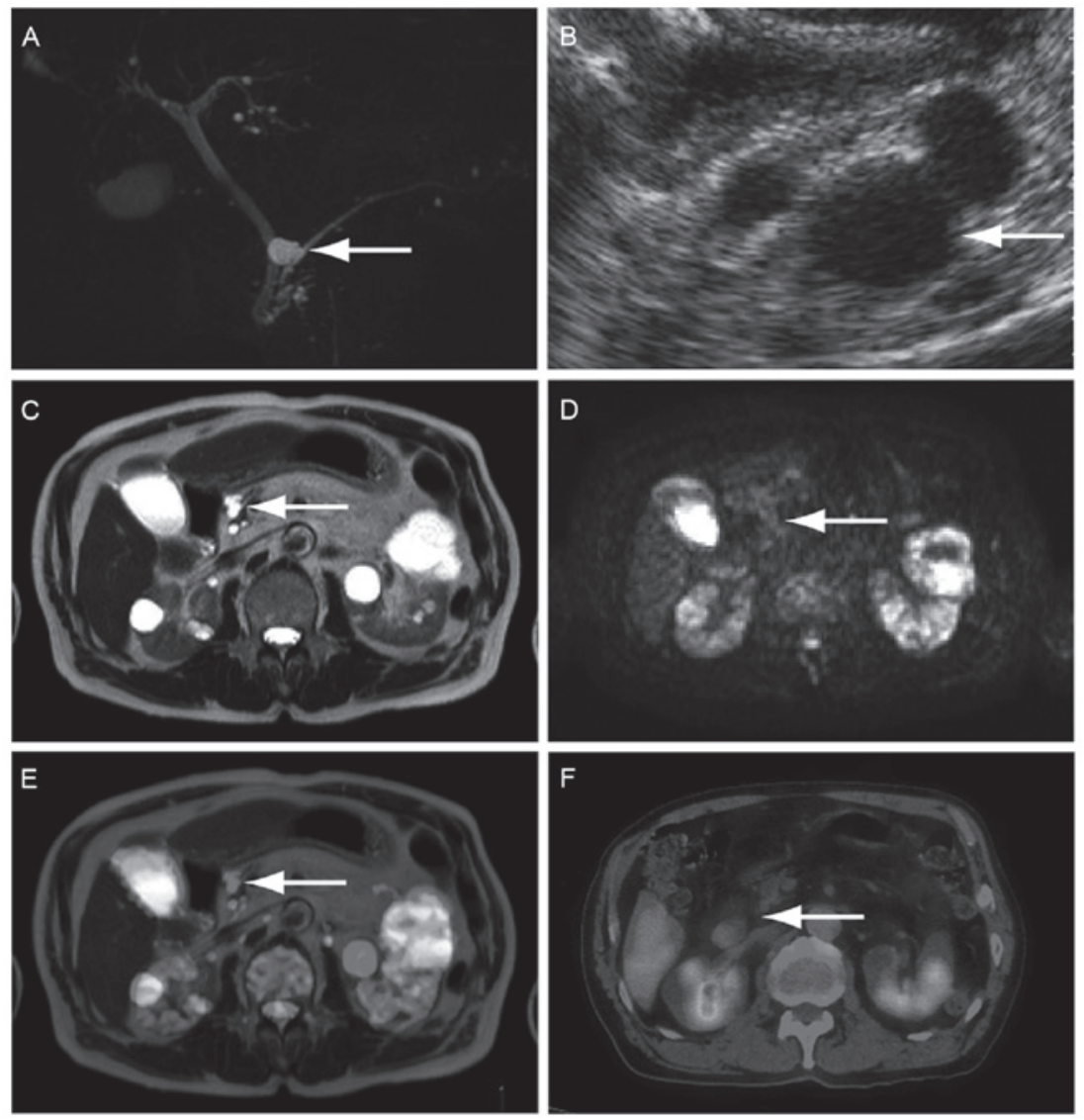

Figure 3. A representative case of intraductal papillary mucinous neoplasm in an 84-year-old man who was regularly followed-up for hepatitis B surface antibody (+). (A) Magnetic resonance cholangiopancreatography shows a cystic lesion (arrow) in the head of the pancreas. (B) Endoscopic US clearly shows a cystic lesion. (C) A T2-weighted image shows a high intensity area in the head of the pancreas. (D) The high intensity area is negative on the DWIBS image. (E) DWIBS/T2 image demonstrates no positive results in the head of the pancreas. (F) PET/CT is also negative for the cystic lesion in the pancreas head. The cystic lesion was finally diagnosed as an intraductal papillary mucinous neoplasm and the patient was followed-up for 30 months, with not evident changes. DWIBS/T2, diffusion-weighted whole body imaging with background body signal suppression/T2-weighted image fusion; PET, positron emission tomography; CT, computed tomography.

Furthermore, the present DWIBS/T2 analysis did not deal with whole body scans, but only evaluated abdominal cavity or pelvis. In the future, more patients should be studied to confirm the present results.
In conclusion, DWIBS/T2 was more found to be sensitive for diagnosing cancer of organs in the abdominal cavity as compared with PET/CT. In addition, negative results with DWIBS/T2 and PET/CT were useful for the diagnosis of 
benign lesions, such as adenomyomatosis of the gallbladder and IPMN.

\section{References}

1. Cameron AM: Screening for viral hepatitis and hepatocellular cancer. Surg Clin North Am 95: 1013-1021, 2015.

2. Clancy TE: Surgery for Pancreatic Cancer. Hematol Oncol Clin North Am 29: 701-716, 2015.

3. Goji T, Kimura T, Miyamoto H, Takehara M, Kagemoto K, Okada Y, Okazaki J, Takaoka Y, Miyamoto Y, Mitsui Y, et al: A phase I/II study of fixed-dose-rate gemcitabine and S-1 with concurrent radiotherapy for locally advanced pancreatic cancer. Cancer Chemother Pharmacol 76: 615-620, 2015.

4. Cid-Arregui A and Juarez V: Perspectives in the treatment of pancreatic adenocarcinoma. World J Gastroenterol 21: 9297-9316, 2015

5. Farrell JJ: Prevalence, diagnosis and management of pancreatic cystic neoplasms: Current status and future directions. Gut Liver 9: 571-589, 2015.

6. Fong ZV and Castillo CF: Intraductal papillary mucinous adenocarcinoma of the pancreas: Clinical outcomes, prognostic factors, and the role of adjuvant therapy. Viszeralmedizin 31 43-46, 2015.

7. Wada K, Takaori K and Traverso LW: Screening for Pancreatic Cancer. Surg Clin North Am 95: 1041-1052, 2015.

8. Vaitheesvaran B, Xu J, Yee J, Q-Y L, Go VL, Xiao GG and Lee WN: The Warburg effect: A balance of flux analysis. Metabolomics 11: 787-796, 2015.

9. Pietryga JA and Morgan DE: Imaging preoperatively for pancreatic adenocarcinoma. J Gastrointest Oncol 6: 343-357, 2015.

10. Dai T, Popa E and Shah MA: The role of ${ }^{18} \mathrm{~F}-\mathrm{FDG}$ PET imaging in upper gastrointestinal malignancies. Curr Treat Options Oncol 15: 351-364, 2014.

11. Kato H, Kuwano H, Nakajima M, Miyazaki T, Yoshikawa M, Ojima H, Tsukada K, Oriuchi N, Inoue T and Endo K: Comparison between positron emission tomography and computed tomography in the use of the assessment of esophageal carcinoma. Cancer 94: 921-928, 2002.

12. Sehy JV, Ackerman JJ and Neil JJ: Apparent diffusion of water, ions and small molecules in the Xenopus oocyte is consistent with Brownian displacement. Magn Reson Med 48: 42-51, 2002.

13. Koike N, Cho A, Nasu K, Seto K, Nagaya S, Ohshima Y and Ohkohchi N: Role of diffusion-weighted magnetic resonance imaging in the differential diagnosis of focal hepatic lesions. World J Gastroenterol 15: 5805-5812, 2009.

14. Takahara T, Imai Y, Yamashita T, Yasuda S, Nasu S and Van Cauteren M: Diffusion weighted whole body imaging with background body signal suppression (DWIBS): Technical improvement using free breathing, STIR and high resolution 3D display. Radiat Med 22: 275-282, 2004.

15. Kwee TC, Takahara T, Ochiai R, Nievelstein RA and Luijten PR Diffusion-weighted whole-body imaging with background body signal suppression (DWIBS): Features and potential applications in oncology. Eur Radiol 18: 1937-1952, 2008.

16. Sommer G, Wiese M, Winter L, Lenz C, Klarhöfer M, Forrer F, Lardinois D and Bremerich J: Preoperative staging of non-small-cell lung cancer: Comparison of whole-body diffusion-weighted magnetic resonance imaging and $18 \mathrm{~F}$-fluorodeoxyglucose-positron emission tomography/computed tomography. Eur Radiol 22: 2859-2867, 2012.
17. Nechifor-Boilă IA, Bancu S, Buruian M, Charlot M, Decaussin-Petrucci M, Krauth JS, Nechifor-Boilă AC and Borda A: Diffusion weighted imaging with background body signal suppression/T2 image fusion in magnetic resonance mammography for breast cancer diagnosis. Chirurgia (Bucur) 108: 199-205, 2013.

18. Wang Y, Miller FH, Chen ZE, Merrick L, Mortele KJ, Hoff FL, Hammond NA, Yaghmai V and Nikolaidis P: Diffusion-weighted MR imaging of solid and cystic lesions of the pancreas. Radiographics 31: E47-E64, 2011.

19. Tanaka S, Ikeda K, Uchiyama K, Iwamoto T, Sanayama Y, Okubo A, Nakagomi D, Takahashi K, Yokota M, Suto A, et al: [18F]FDG uptake in proximal muscles assessed by PET/CT reflects both global and local muscular inflammation and provides useful information in the management of patients with polymyositis/dermatomyositis. Rheumatology (Oxford) 52: 1271-1278, 2013.

20. Grabinska K, Pelak M, Wydmanski J, Tukiendorf A and d'Amico A: Prognostic value and clinical correlations of 18-fluorodeoxyglucose metabolism quantifiers in gastric cancer. World J Gastroenterol 21: 5901-5909, 2015.

21. Manenti G, Cicciò C, Squillaci E, Strigari L, Calabria F, Danieli R, Schillaci O and Simonetti G: Role of combined DWIBS/3D-CE-T1w whole-body MRI in tumor staging: Comparison with PET-CT. Eur J Radiol 81: 1917-1925, 2012.

22. Wang N, Zhang M, Sun T, Chen H, Huang Z, Yan L, Wang L, Li F and Chen Z: A comparative study: diffusion weighted whole body imaging with background body signal suppression and hybrid Positron Emission Computed Tomography on detecting lesions in oncologic clinics. Eur J Radiol 81: 1662-1666, 2012.

23. Chen JL, Appelbaum DE, Kocherginsky M, Cowey CL, Rathmell WK, McDermott DF and Stadler WM: FDG-PET as a predictive biomarker for therapy with everolimus in metastatic renal cell cancer. Cancer Med 2: 545-552, 2013.

24. Yamada A, Oguchi K, Fukushima M, Imai Y and Kadoya M: Evaluation of 2-deoxy-2-[18F]fluoro-D-glucose positron emission tomography in gastric carcinoma: Relation to histological subtypes, depth of tumor invasion, and glucose transporter-1 expression. Ann Nucl Med 20: 597-604, 2006.

25. Yun M: Imaging of gastric cancer metabolism using 18 F-FDG PET/CT. J Gastric Cancer 14: 1-6, 2014.

26. Cafagna D, Rubini G, Iuele F, Maggialetti N, Notaristefano A, Pinto D, Niccoli-Asabella A, Palmiotti G, Lasciarrea M and Maggialetti A: Whole-body MR-DWIBS vs. [18F]-FDG-PET/CT in the study of malignant tumors: A retrospective study. Radiol Med 117: 293-311, 2012

27. Joo I, Lee JY, Kim JH, Kim SJ, Kim MA, Han JK and Choi BI: Differentiation of adenomyomatosis of the gallbladder from early-stage, wall-thickening-type gallbladder cancer using high-resolution ultrasound. Eur Radiol 23: 730-738, 2013.

28. Tomizawa M, Shinozaki F, Motoyoshi Y, Sugiyama T, Yamamoto $\mathrm{S}$ and Ishige N: Diffusion-weighted whole body imaging with background body signal suppression/T2 image fusion is negative for patients with intraductal papillary mucinous neoplasm. Hepatogastroenterology 62: 463-465, 2015.

29. Ferrari C, Minoia C, Asabella AN, Nicoletti A, Altini C, Antonica F, Ficco M, Guarini A, Maggialetti N and Rubini G: Whole body magnetic resonance with diffusion weighted sequence with body signal suppression compared to (18)F-FDG PET/CT in newly diagnosed lymphoma. Hell J Nucl Med 17 (Suppl 1): S40-S49, 2014. 